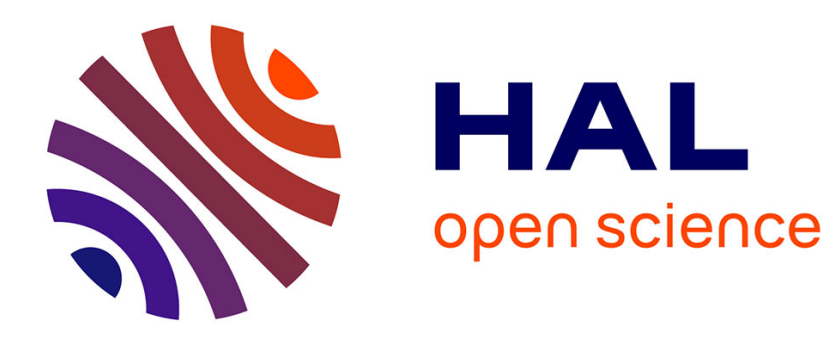

\title{
Efficacité des compteurs à rayons $\gamma$
}

Nicolas C.M. Marty

\section{To cite this version:}

Nicolas C.M. Marty. Efficacité des compteurs à rayons $\gamma$. J. Phys. Radium, 1947, 8 (1), pp.29-32. 10.1051/jphysrad:019470080102900 . jpa-00234016

\section{HAL Id: jpa-00234016 https://hal.science/jpa-00234016}

Submitted on 1 Jan 1947

HAL is a multi-disciplinary open access archive for the deposit and dissemination of scientific research documents, whether they are published or not. The documents may come from teaching and research institutions in France or abroad, or from public or private research centers.
L'archive ouverte pluridisciplinaire HAL, est destinée au dépôt et à la diffusion de documents scientifiques de niveau recherche, publiés ou non, émanant des établissements d'enseignement et de recherche français ou étrangers, des laboratoires publics ou privés. 


\title{
EFFIGAGITÉ DES GOMPTEURS A RAYONS $\gamma$
}

\author{
Par Mme N. MARTY. \\ Laboratoire de Chimie nucléaire du Collège de France.
}

\begin{abstract}
Sommaire. - Après un rappel de différents travaux relatifs à l'efflcacité des compteurs à rayons $\gamma$, on détermine l'épaisseur optima des compteurs pour les différentes valeurs de l'énergie des $\gamma$. On indique une méthode de calcul des rendements des compteurs tenant compte des différents effets (photoélectrique, Compton, matérialisation). On donne les résultats numériques pour des $\gamma$ de 0,2 à $5 \mathrm{MeV}$ et des compteurs de cuivre et d'aluminium. On a effectué une vérification expérimentale de ces résultats avec une source de Ra en équilibre avec ses dérivés et un compteur de cuivre. Les résultats théoriques et expérimentaux concordent à io pour 100 près.
\end{abstract}

Introduction. - La connaissance de l'efficacité ou rendement d'un compteur à rayons $\gamma$ présente un grand intérêt; elle permet, par exemple, de déterminer le nombre de quanta d'une énergie donnée émis dans une désintégration donnée, elle intervient aussi constamment dans la méthode de mesures en coïncidences (coïncidences $\beta \gamma$ et $\gamma \gamma$ ).

Plusieurs travaux ont été faits à ce sujet; le premier, en 1936 , Von Droste [r] signala que l'efficacité d'un compteur à rayons $\gamma$ dépendait de l'énergie des $\gamma$ utilisés, il détermina expérimentalement le rendement d'un compteur en laiton de $\mathrm{I} \mathrm{mm}$ d'épaisseur de paroi pour les rayons $\gamma$ de Th $C^{\prime \prime}$ de $2,7 \mathrm{MeV}$ et ceux de $\operatorname{Th} B$ de $0,24 \mathrm{MeV}$, et calcula l'aspect de la courbe efficacité-énergie des $\gamma$ à l'aide de considérations théoriques. Yukawa et Sakata [2], se plaçant à un point de vue uniquement théorique, calculèrent les efficacités relatives de compteurs de plomb et d'aluminium pour des énergies allant de 0,2 à ı $\mathrm{MeV}$. Norling [3], après avoir précisé les formules théoriques générales donnant le rendement d'un compteur à rayons $\gamma$ dans le cas où la source est à faible distance $d u$ compteur, formules trop compliquées pour être calculées, détermina expérimentalement l'efficacité de compteurs d'aluminium, cuivre et plomb pour des $\gamma$ de différentes énergies.

Nous avons repris le problème en utilisant une méthode déjà indiquée [4] et nous avons calculé l'efficacité de compteurs d'aluminium et de cuivre pour des énergies de 0,2 à $5 \mathrm{MeV}$, chacun d'eux ayant l'épaisseur de paroi optima pour l'énergie considérée. Nous avons fait une vérification expérimentale de l'ordre de grandeur des résultats obtenus.

Définition de l'efficacité d'un compteur à rayons $\gamma$. - Soit une source située à l'infini, envoyant sur l'ensemble du compteur un faisceau de rayons $\gamma$ parallèles et normaux à l'axe du compteur, nous appellerons efficacité du compteur pour une énergie donnée des $\gamma$ incidents, le rapport du nombre de décharges au compteur au nombre de quanta tombant sur la surface effective $S$ du compteur : pour un compteur cylindrique de diamètre extérieur $D$ et de longueur $h$ (la distance séparant les bouchons du compteur) $S=h D$ (fig. I).

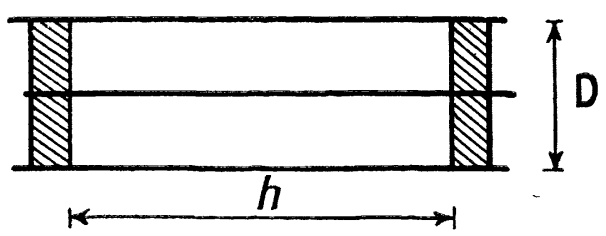

Fig. I.

Le rendement est fonction de l'énergie des $\gamma$ incidents et de la matière du compteur, il peut être considéré comme indépendant du rapport $\frac{h}{D}$.

Mécanisme de la décharge. - Un rayon $\gamma$, absorbé dans la paroi du compteur, donne en proportion variant suivant son énergie, des photoélectrons, des électrons Compton ou des électrons de matérialisation. Si le parcours effectif de ces électrons est supérieur à la distance qui sépare leur lieu de formation de l'intérieur du compteur, ils peuvent pénétrer dans l'atmosphère gazeuse du compteur et produisent une décharge. Dans tout ce travail, nous utiliserons la relation liant le parcours à l'énergie des électrons, relation relativement bien connue, surtout pour le parcoùrs dans i'aluminium à la suite des travaux de Varder [5], Madgwick [6] et Eddy [7], en extrapolant jusqu'à $5 \mathrm{MeV}$ la courbe de Madgwick. Cette notion de parcours n'est vraie qu'en moyenne pour un grand nombre d'électrons. Par raison de symétrie, nous considérerons qu'un électron 
émis dans une direction a son parcours dans cette direction.

Épaisseur des parois du compteur. - L'épaisseur optima d'un compteur pour des rayons $\gamma$ d'énergie déterminée est égale au parcours dans le métal formant la paroi du compteur des électrons les plus énergiques. Avec une telle épaisseur de paroi, seuls interviennent les électrons issus du compteur et l'on peut alors les évaluer.

Tableau 1.

\begin{tabular}{|c|c|c|c|c|c|c|}
\hline & \multicolumn{6}{|c|}{ Énergie des $\gamma$ incidents (MeV). } \\
\hline & $0,2$. & $\mathbf{0 , 5}$ & 1. & $\mathbf{2 .}$ & $\mathbf{3 , 5 .}$ & $\mathbf{5}$ \\
\hline $\begin{array}{r}\text { Épais se ur } \\
\text { optima (en } \\
\text { cm) : } \\
\text { de Al... } \\
\text { Cu... }\end{array}$ & $0,0,0048$ & 0,064 & 0,19 & 0,3 & 0,6 & 0,88 \\
& & & & & & \\
\hline
\end{tabular}

Nous calculerons l'efficacité de compteurs de cuivre et d'aluminium pour des rayons $\gamma$ incidents de $0,2,0,5,1,2,3,5,5 \mathrm{MeV}$, les épaisseurs correspondantes des parois étant données dans le Tableau $\mathrm{I}$. Les calculs ne sont valables que si le compteur a au moins l'épaisseur indiquée, toute épaisseur supplémentaire correspond à une absorption du faisceau des $\gamma$ incidents, ce qui diminue légèrement l'efficacité du compteur : par exemple, si l'on utilise un compteur de $\mathrm{Cu}$ de $1,2 \mathrm{~mm}$ d'épaisseur correspondant à des $\gamma$ de $2,5 \mathrm{MeV}$ pour des $\gamma$ d'une énergie plus faible, les efficacités calculées ci-dessous sont diminuées de I 4 pour roo pour o,5 MeV, 8 pour Ioo pour $\mathrm{I} \mathrm{MeV}$ et 2 pour 1 oo pour $2 \mathrm{MeV}$.

Étude des différents effets. - 1. Effet photoélectrigue. - Les coefficients d'absorption photoélectrique $\mu_{p}$ sont donnés en fonction de l'énergie $h \nu$ des $\gamma$ incidents par les courbes de Hulme et Sauter.

L'énergie des électrons émis est $h \nu-E_{k} \sim h \nu$, $E_{k}$ étant l'énergie du niveau $K$ du métal constituant le compteur.

L'intensité $I(\theta)$ des électrons émis dans la direction faisant l'angle $\theta$ avec la direction du faisceau incident est

$$
I(\theta)=\frac{\sin ^{2} \theta}{(I-\beta \cos \theta)^{4}} \quad[8] .
$$

Les électrons étant tous émis vers l'avant, on ne considérera que la moitié antérieure du compteur et, par symétrie, on effectuera tous les calculs pour un seul quadrant.

Étant donnée la forme cylindrique du compteur, le parcours des électrons dépend de la direction de projection et de l'endroit où est absorbé le quantum : la paroi du compteur sera divisée en un certain nombre de couches concentriques et en neuf secteurs, l'élément $i j$ correspondra au secteur $i$ et à la couche $j$ (fig. 2).

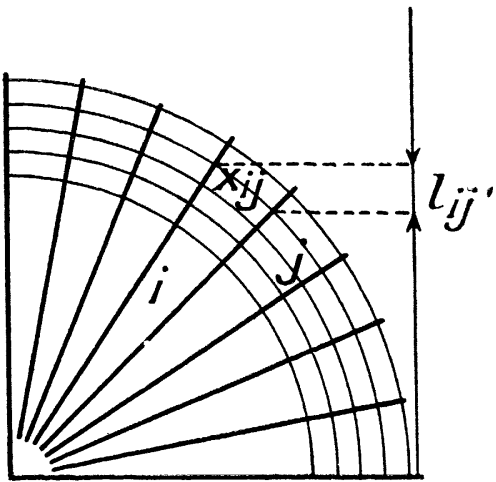

Fig. 2.

Soit $m$ le nombre de quanta tombant sur la surface effective $h D$ du compteur, par centimètre carré, il en arrive $m^{\prime}=\frac{m}{h \mathrm{D}}$, après la traversée d'une épaisseur $x$ de matière $m^{\prime}\left(\mathrm{I}-e^{-\mu_{p} x}\right)$ quanta sont absorbés, chaque quantum arrache un électron d'énergie $h \nu$, de ces $\mathrm{m}^{\prime}\left(\mathrm{r}-e^{-u_{p} x}\right)$ électrons formés

$$
k_{p} m^{\prime}\left(\mathrm{I}-e^{-u_{p} \cdot x}\right) \sim k_{p} m^{\prime} \mu_{p} x
$$

pénètrent à l'intérieur du compteur; $k$ dépend du lieu de formation de l'électron et de sa direction d'émission.

Calcul de k. - On fera le calcul de $k$ pour le point milieu de chaque élément $i j$ en se plaçant dans un plan axial et dans un plan radial perpendiculaire à l'axe du cylindre et l'on prendra la moyenne de ces valeurs. Considéróns l'élément $i j$, d'après la relation parcours-énergie, peuvent pénétrer dans le compteur les électrons émis entre les directions formant les angles $\theta_{1}$ et $\theta_{2}$ avec la direction du faisceau incident, on calculera l'absorption de ces électrons en utilisant les courbes de Madgwick et Eddy [6], [7] donnant le nombre d'électrons transmis après traversée d'une épaisseur donnée de matière; la loi de distribution angulaire des photoélectrons donne le pourcentage des électrons émis entre les directions $\theta_{1}$ et $\theta_{2}$ et permet ainsi de déduire $k_{i j}$.

Le nombre de quanta tombant sur l'élément ij est $h l_{i j} m^{\prime}$, d'après les mesures de Jorgensen [9], on peut considérer que, pour des compteurs suffisamment étroits, toute la longueur $h$ intervient. Dans cet élément, $h l_{i j} m^{\prime} \mu_{p} x_{i j}$ électrons sont formés et $h l_{i j} m^{\prime} \mu_{p} x_{i j} k_{i j}$ électrons pénètrent dans le compteur; 
pour l'ensemble des deux quadrants on a

$$
n_{\mu}=2 h m^{\prime} \mu_{p} \sum_{i} \sum_{j} l_{i j} k_{i j} x_{i j}=2 \frac{m}{D} \mu_{\mu} \sum_{i} \sum_{j} l_{i j} k_{i j} x_{i j}
$$

électrons qui pénètrent dans le compteur et déterminent une décharge.

Si la paroi du compteur est peu épaisse, $l_{i}$ peut être considéré comme constant pour toutes les valeurs de $j$ : on a alors

$$
n_{p}=\frac{2 m}{D} \mu_{p} \sum_{i} l_{i} \sum_{j} k_{i j} x_{i j}
$$

2. Effet Compton. - Le coefficient d'absorption $\mu_{c}$ est calculé d'après la formule de Klein et Nishina [1 o], l'énergie des électrons projetés sous un angle $\theta$ est

$$
W=\frac{2 \alpha h \nu}{\mathrm{I}+2 \alpha+(\mathrm{I}+\alpha)^{2} \operatorname{tg}^{2} \theta} \quad \text { avec } \quad \alpha=\frac{h \nu}{m c^{2}} .
$$

La distribution angulaire des électrons est donnée par la formule de Klein et-Nishina et les courbes de Kahan et Skobelzyn [io], [i I].

D'après un raisonnement semblable à celui effectué à propos des photoélectrons, le nombre d'électrons Compton qui pénètrent à l'intérieur du compteur est

$$
n_{c}=2 \frac{m}{D} \mu_{c} \sum_{i j} k_{i j} l_{i j} x_{i j}
$$

Seul diffère le calcul de $k_{i j}$.

A chaque direction de projection des électrons correspond une valeur de l'énergie donc du parcours de ces électrons. On déterminera par approximations successives la valeur maxima de l'angle $\theta$ telle que l'électron émis dans cette direction puisse pénétrer à l'intérieur du compteur, ceci en se plaçant comme précédemment successivement dans un plan radial et dans un plan diamétral et en considérant les électrons émis de part et d'autre de la direction des $\gamma$ incidents : leur parcours, en effet, diffère en raison de la courbure de la paroi du compteur. L'absorption des électrons sera calculée comme dans le cas précédent.

3. Effet de matérialisation. - Cet effet n'existe que pour des quanta d'énergie supérieure à $\mathrm{r} M \mathrm{MeV}$.

Le coefficient d'absorption $\mu_{m}$ est calculé d'après les courbes de Bethe et Heitler [12]. D'après Heitler (Quantum theory of Radiation) pour des $\gamma$ d'énergie inférieure à ro $\mathrm{MeV}$, les électrons positifs et négatifs sont émis avec la même énergie $\mathrm{W}=\frac{h v-2 m_{0} c^{2}}{2}$ dans un cône d'angle $\theta=\frac{m c^{2}}{h \nu}$. Excepté le cas des rayons $\gamma$ de $2 \mathrm{MeV}$ où l'effet de matérialisation est très faible, tous les électrons sont émis dans un angle inférieur à $10^{\circ}$; on considérera qu'ils sont projetés dans la direction des rayons $\gamma$ incidents.
Pour chaque secteur, les électrons qui ont tous la même énergie peuvent pénétrer dans le compteur s'ils sont formés à une distance de l'intérieur du compteur inférieure à l'épaisseur correspondant à leur parcours $x_{r j}$ dans un plan radial, $x_{a}$ dans un plan axial, d'où

$$
n_{m}=2 \times 2 \frac{m}{D} \mu_{m} \sum_{j} l_{j} k_{j} \frac{x_{a}+x_{r j}}{2} .
$$

Le facteur 2 tient compte du fait qu'il y a deux électrons projetés par quantum; $k_{j}$ dépend de l'absorption des électrons formés.

Résultats. - On a calculé l'efficacité de compteurs de $1,7 \mathrm{~cm}$ de diamètre intérieur, les épaisseurs des parois sont données par le Tableau I.

La précision des données théoriques utilisées (distribution angulaire des électrons en particulier) et surtout de la relation parcours-énergie et de l'absorption des électrons ne permet pas d'obtenir des résultats à plus de ıo pour 100 près, les résultats

\begin{tabular}{|c|c|c|c|c|}
\hline $\begin{array}{c}\text { Énergie des } \gamma \\
(\mathrm{MeV}) .\end{array}$ & $n_{p} .10^{2}$ & $n_{c} .10^{2}$ & $n_{m} \cdot 10^{2}$. & e. $10^{2}$ \\
\hline \multicolumn{5}{|c|}{ Compteurs d'Al. } \\
\hline$o, 2 \ldots \ldots \ldots$ & 0,009 & $o, 046$ & - & 0,055 \\
\hline$o, \check{\jmath} \ldots \ldots$ & 0,002 & 0,45 & - & 0,45 \\
\hline $\mathbf{1} \ldots \ldots$ & - & 0,77 & - & 0,77 \\
\hline $2 \ldots \ldots \ldots$ & - & I , 28 & 0,02 & I, 3 \\
\hline $3, j \ldots \ldots$ & - & I, 8 & 0,12 & I, 92 \\
\hline $5 \ldots \ldots \ldots$ & - & 2,25 & I, 3 & 2,55 \\
\hline \multicolumn{5}{|c|}{ Compteurs de $\mathrm{Gu}$. } \\
\hline $0,2 \ldots, \ldots$ & 0,15 & 0,04 & - & $o$, I 9 \\
\hline $0,5 \ldots \ldots$ & $o, 03$ & 0,29 & - & 0,32 \\
\hline I $\ldots \ldots \ldots$ & - & $o, 69$ & - & 0,69 \\
\hline $2 \ldots \ldots \ldots$ & - & I, 26 & $o, 03$ & I , 29 \\
\hline $3, j \ldots \ldots$ & - & 2,07 & 0,3 & 2,37 \\
\hline $5 \ldots \ldots \ldots$ & - & 2,6 & 0,7 & 3,3 \\
\hline
\end{tabular}
pouvant être considérés comme plus précis dans le cas des compteurs d'aluminium et des énergies plus faibles (jusqu'à $3 \mathrm{MeV}$ environ).

Tableau II.

Remarque. - Les résultats obtenus pour un compteur de $1,7 \mathrm{~cm}$ de diamètre intérieur peuvent être considérés comme valables pour des diamètres sensiblement différents. Nous avons effectué des calculs analogues pour des compteurs d'aluminium de $2,2 \mathrm{~cm}$ et de $1,2 \mathrm{~cm}$ de diamètre intérieur, et des $\gamma$ incidents de $\mathrm{I} \mathrm{MeV}$; les résultats obtenus diffèrent de moins de 5 pour ıoo de celui donné dans le Tableau II pour cette énergie. 
Vérification expérimentale. - La vérification précise de ces calculs nécessiterait la connaissance de corps émetteurs d'un seul rayonnement $\gamma$ d'énergie et d'intensité bien déterminées, ces énergies s'étageant de quelques centaines de mille à quelques millions d'électronvolts. Nous avons fait une vérification de l'ordre de grandeur des valeurs obtenues pour les efficacités d'après une méthode utilisée par Savel [13] dans le cas d'une chambre d'ionisation, en nous servant d'une source de 2,2 I $\mathrm{mg}$ de Ra en équilibre avec ses dérivés; d'après Ellis [14] et Skobeltzyn, les énergies et les intensités en quanta par désintégration pour les raies principales sont :

$\operatorname{Ra} B$ :

\begin{tabular}{|c|c|c|c|c|}
\hline \multirow{2}{*}{$\begin{array}{l}\text { Energie }(\mathrm{keV}) \ldots \\
\text { Intensités....... }\end{array}$} & 240,6 & $29^{3}, 7$ & 349,9 & - \\
\hline & 0,115 & 0,258 & 0,450 & - \\
\hline \multicolumn{5}{|l|}{$\operatorname{Ra} C:$} \\
\hline Énergie (keV). & 607 & 766 & $9^{33}$ & I 120 \\
\hline & I 238 & 1379 & $176 \mathrm{I}$ & $219^{3}$ \\
\hline tensit & $o, 658$ & 0,065 & 0,067 & 0,206 \\
\hline & 0,0 & 0,064 & 0,258 & 0,074 \\
\hline
\end{tabular}

La source était placée à $77 \mathrm{~cm}$ d'un compteur en cuivre d'épaisseur 1,2 $\mathrm{mm}$ et de hauteur $4,3 \mathrm{~cm}$, on interposait des écrans de plomb entre la source et le compteur et l'on déterminait le nombre d'impulsions au compteur par minute. D'autre part, on calculait, en utilisant les valeurs d'efficacité données par le Tableau II corrigées si l'épaisseur du compteur était supérieure à l'épaisseur utilisée dans le calcul, le nombre d'électrons, donc de décharges au compteur pour l'ensemble des raies et les épaisseurs d'écran utilisées.

Résultats.

\begin{tabular}{|c|c|c|c|c|}
\hline Écrans $\mathrm{Pb}(\mathrm{en} \mathbf{c m})$. & $\left\{\begin{array}{l}7 \\
4,5\end{array}\right.$ & $\begin{array}{l}6,5 \\
3,9\end{array}$ & $\begin{array}{l}6 \\
3,3\end{array}$ & $\begin{array}{l}5,5 \\
2,7\end{array}$ \\
\hline Valeurs mesurées. & $\left\{\begin{array}{r}71 \\
271\end{array}\right.$ & $\begin{array}{r}9^{5} \\
370\end{array}$ & $\begin{array}{l}117 \\
498\end{array}$ & $\begin{array}{l}159 \\
719\end{array}$ \\
\hline calculé & & $\begin{array}{r}84 \\
363\end{array}$ & $\begin{array}{l}\text { I } 13 \\
5 \text { I0 }\end{array}$ & $\begin{array}{l}145 \\
728\end{array}$ \\
\hline
\end{tabular}

Les valeurs calculées concordent à ro pour roo avec les résultats expérimentaux. Ceci ne peut que confirmer les valeurs des efficacités calculées pour des énergies de l'ordre de 1 à $2 . \mathrm{MeV}$; en effet, les raies $\gamma$ d'énergies plus faibles interviennent peu du fait de leur absorption plus grande dans les écrans de plomb et de l'efficacité plus faible du compteur pour ces énergies.

Conclusion. - L'accord est satisfaisant entre les résultats expérimentaux et théoriques. On ne peut compter obtenir des valeurs théoriques beaucoup plus précises pour les efficacités des compteurs à rayons $\gamma$ en raison du grand nombre de phénomènes assez mal déterminés qui interviennent et surtout de la difficulté que l'on a à préciser les notions de parcours et d'absorption des électrons dans la matière.

Je tiens à remercier le Professeur Joliot pour l'intérêt qu'il a bien voulu prendre à ce travail et les conseils qu'il m'a donnés à ce sujet.

BIBLIOGRAPHIE.

[i] Van Droste, Z. f. Physik, r 936,100,p. 529 et 104, p. 474. [2] Yukawa et Sakata, Sc. P. I. of Phys, and Chem. Res., I 937,31, p. 187 .

[3] Norling, Archiv. för mat. Astronomisk Fysik, Bd.27 A, $\mathrm{n}^{\circ} 27$.

[4] N. Marty, J. Physique et Rad., I $944,7^{\mathrm{e}}$ série, 5, p. 276.

[5] Varder, Phil. Mag., i 9 I 5, 29, p. 725.

[6] Madgwick, Proc. Camb. Phil. Soc., i 927,23 , p. 970.
[7] EDDy, Ibid., r929, 25, p. 50 .

[8] Sauter, Ann. der Physik, 1931, 11, p. 477.

[9] JoRgensen, Rev. scient. Instr., 1939,10, p. 34.

[i o] Kahan, J. Physique et Rad., i 939,10 , p. 430.

[i I] Skobeltzyn, Z. Physik, r 930,65, p. 784.

[1 2] Bethe et Heitler, Proc. Roy. Soc., 1934, 146, p. I09.

[13] Savel, Cah. Phys., i 944, no 19, p. 39.

[ 14] Ellis, Proc. Roy. Soc., i 934,143, p. 350. 